\title{
Topology Matters: Network topology affects outcomes from community ecology
} neutral models

\author{
Denis White \\ US Environmental Protection Agency \\ 200 SW $35^{\text {th }}$ Street \\ Corvallis, Oregon, 97333 USA \\ Email: white.denis@epa.gov \\ Phone: 541.754 .4476 \\ Fax: $\quad 541.754 .4799$
A. Ross Kiester
Biodiversity Futures Consulting
5550 SW Redtop
Corvallis, OR 97333

Date of this version: 2 November 2007

Accepted for publication in Computers, Environment and Urban Systems 


\begin{abstract}
Topology affects outcomes of processes in planar networks. Hexagon tessellations have different performance than, and are often superior to, square tessellations in applications such as fluid dynamics, percolation theory, self-avoiding walks, survey sample design, and quantization. Hexagon tessellations and square tessellations using both von Neumann and Moore neighborhoods were examined as a network topology for neutral ecology community models, following the simulation approach of Graham Bell. These models, which assume identical life history and movement properties for each individual of each species, produce collective properties of communities, such as abundance distributions, range distributions, spatial variation in abundance, species-area curves, and spatial variation in species composition, that match many empirical patterns. The simulations in this study varied the dispersal rate but kept birth, death, and immigration rates constant. For these experiments, ending community populations, species richness, Shannon diversity, and Simpson diversity were clearly different for the different topologies, but the relationship between the topologies varied as the dispersal rate changed. Empirical distributions of the performance measures also showed clear differences among topologies. The interaction of topology with dispersal, spatial boundary effects, and other parameters of these models appears to be quite complex and warrants further research.
\end{abstract}

Key Words: neutral model, community ecology, topology, simulation model, population biology 


\section{Introduction}

Models of spatial processes in two dimensions often use one of the only three regular tessellations in the plane: squares, hexagons, or triangles. Triangles are seldom used, hexagons infrequently, and the vast majority of regular grid applications uses squares. While square tessellations are easy to use there is one significant problem. Hexagons have six neighbors each with the same border length (Figure 1a). Squares have four orthogonal neighbors each with the same border length and four diagonal neighbors adjacent only at a vertex. The orthogonal neighbors are called the von Neumann neighborhood (Figure 1b), and those plus the diagonal neighbors are called the Moore neighborhood (Figure 1c).

Using the von Neumann neighborhood neglects the close proximity of the diagonal neighbors, but using the Moore neighborhood implicitly assumes, in effect, that the cell is an octagon and octagons do not tile the plane. Of course, the diagonal neighbors can be weighted (see Birch 2006 for an example), but it is not obvious what the weighting should be, depending on whether the process assumes locations are points or areas. An alternative used in some cellular automata applications is the Margolus neighborhood, which can be visualized as the six closest neighbors in a tilted square tessellation (Figure 1d). The Margolus neighborhood is implemented with two 4x4-cell blocked square tessellations which are offset from one another by one row and one column. The Margolus neighborhood then consists of a cell and its three block neighbors in one tessellation plus its three block neighbors in the other tessellation. This procedure essentially simulates hexagonal adjacency relations.

The motivation for examining this issue in the context of community ecology population models comes from a wide literature that describes the differential performance of alternative topologies on a number of physical properties and processes when represented in a plane.

The most important of the findings on topological effects is probably in the simulation of the Navier-Stokes equations for fluid dynamics in two dimensions. Both analytical results and simulations show not only differences between square and hexagonal tessellations, but also that the square tessellation (using the von Neumann neighborhood) has an anisotropic viscosity that is an artifact resulting, apparently, from the ambiguity in connectivity between adjacent side neighbors and adjacent vertex neighbors (Toffoli and Margolus 1987 p. 178, Frisch et al. 1986). The Navier-Stokes results raise a question for ecology: is particle movement such as that found in ecological processes subject to similar artifactual effects when implemented in a square tessellation?

Another finding is from analysis of the percolation movement model, or graph connectivity property, in two (and greater) dimensions. This model has a critical parameter that is topology dependent. This parameter is the threshold of density of occupancy beyond which it is guaranteed that a path will exist from one boundary of the study area to another, connecting occupied locations. If the locations are conceived of as vertices, the model is called site percolation, and if edges, bond percolation. For both types of percolation the critical value for a hexagon tessellation (considered as a triangular lattice representing the movement graph) is less 
than that for a square tessellation (using the von Neumann neighborhood) (Stauffer and Aharony 1992, Wolfram Mathworld 2006).

In geostatistics, a hexagonal tessellation of sampling sites has a lower minimum average standard error, a lower maximum standard error, a larger screen effect (ability of sample points to minimize influence of points farther from the estimation point), and a smaller number of nearest neighbors for stable kriging, than a square tessellation (or any other pattern of sample points) (Olea 1984).

In the quantization of continuous or highly detailed discrete data into chunks or bins more suitable for data analysis or graphical display, hexagons are the optimal solution in two dimensions (Conway and Sloane 1999).

Recent work questions simulations using square tessellations (and other types) and quantifies some of the problems. Flache and Hegselman (2001) found that in agent-based social cooperation simulations, actors will migrate in search of other cooperating actors in hexagon tessellations at a higher rate than in square tessellations, and it takes approximately six times as long for the rates to stabilize. Cioffi-Revilla (2002) points out that the average number of territorial neighbors in political systems is close to six, that is, close to the number of hexagon neighbors, and that the use square tessellations in agent-based model simulations may affect model behavior.

Birch (2006) compared a hexagonal tessellation with a modified square tessellation where diagonal interactions were given half the weight of orthogonal interactions, in an attempt to approximate the behavior of the equiprobable interactions in the hexagonal tessellation. Using a weed spread algorithm where the probability of spread was equal to the proportion of neighbor cells occupied minus the probability that external events (e.g., grazing) would occur, the modified square tessellation and hexagonal tessellation had similar behavior except when the grazing probability was in the range 0.25 to 0.3 . (At 0.25 the square tessellation with only orthogonal interactions [i.e., using the von Neumann neighborhood] does not propagate occupancy to neighbor cells.) Bithell and Macmillan (2007) explored alternatives to regular tessellations with one individual per cell for cellular automata applications in population dynamics. They advocated for a "particle-in-cell" representation where more than one individual can occupy a cell.

Although hexagonal tessellations have been used in ecological modeling at least as far back as 1967 (Murray 1967), the first comparison of tessellation properties for ecological research may have been that of Keller et al. (1979). These authors compared hexagonal and square tessellations for use in evaluating habitat edge effects, spatial trends in habitat distribution, and modeling territory size for terrestrial wildlife.

In this paper the question is posed whether the topology of a network of ecological communities affects the dispersal statistics of populations in the communities. To do this we use a recently proposed theory that does, in some circumstances, appear to produce, when simulated or analyzed mathematically, observed empirical distribution patterns of species and their 
abundances. This theory has been called the neutral theory of community ecology (e.g., Bell 2005) or, alternatively, the unified neutral theory of biodiversity and biogeography (Hubbell 2001).

The theory is neutral in the sense that each species in a community of species that compete with one another is assumed to have the same per capita birth and death rates. This assumption contrasts with most accepted biological knowledge especially when carefully analyzed empirically (Chave 2004). Furthermore, the theory has been developed with the assertion, although not specified as an assumption, of a uniform environment. This limitation, obvious to anyone, has been explicitly investigated by Bell (2005).

What, then, is striking about such a simplified model, having only a handful of parameters, that will be described shortly, is that both analytical results and simulated experiments yield patterns that conform to many of the documented relationships about species, their abundances, and ranges as discussed above. As a number of reviewers have noted (e.g., Brown 2001, Chave 2004), although the theory is almost surely incorrect in its assumptions, it is the most ambitious, creative, and successful attempt so far to account for many of the issues and assemblage data of community ecology. This theory has also, appropriately to its importance, generated substantial investigations both theoretical and empirical into its properties and validity (e.g., Adler 2004, Bell 2003, Chase 2005, Chave et al. 2002, Condit et al. 2002, Enquist et al. 2002, Etienne and Olff 2004, Gilbert and Lechowicz 2004, He 2005, Hubbell 2005, Magurran 2005, McGill 2003, McGill and Collins 2003, McKane et al. 2004, Nee 2005, Nee and Stone 2003, Volkov et al. 2003, Yu et al. 1998).

The present paper contributes to this literature by examining the extent that simulation models of the theory may depend upon, in addition to any other parameters, the geometric nature of the network in which the communities of species are presumed to inhabit.

\section{Methods}

The six parameters for the Bell (2001) version of neutral community ecology models are (1) the number of species in the species pool, (2) the (maximum) number of individuals in a community (a carrying capacity), (3) the probability that an individual of any species will give birth in each time step, (4) the probability that an individual of any species will die in each time step, (5) the probability that a newborn individual will disperse to an adjacent community, and (6) the probability that a new individual of each species will immigrate (from outside the metacommunity) in each time step. It appears from Bell (2005, Figure 1) that he used a square tessellation in his simulations, although this was not specified in his papers nor whether he used the von Neumann or Moore neighborhoods. (Hubbell (2001) used the square tessellation with the Moore neighborhood for simulation results.)

In this study simulation runs were initialized by assigning to each community an initial population of half the carrying capacity randomly distributed to each species. The actions in each time step were immigration, birth and dispersal, death, and maintenance of carrying capacity. Each community was assigned immigrant individuals according to the immigration 
probability, distributed randomly to each species. Each existing individual of each species in each community produced a new individual with probability equal to the probability of birth. Each new individual moved to a random adjacent location with probability equal to the probability of dispersal and continued moving until the probability of dispersal was not met (Bell 2001). Each existing individual died with probability equal to the probability of death. Finally, if the number of individuals in a community exceeded the carrying capacity, the excess were deleted randomly. The algorithm for the neutral model represented in pseudo-code is:

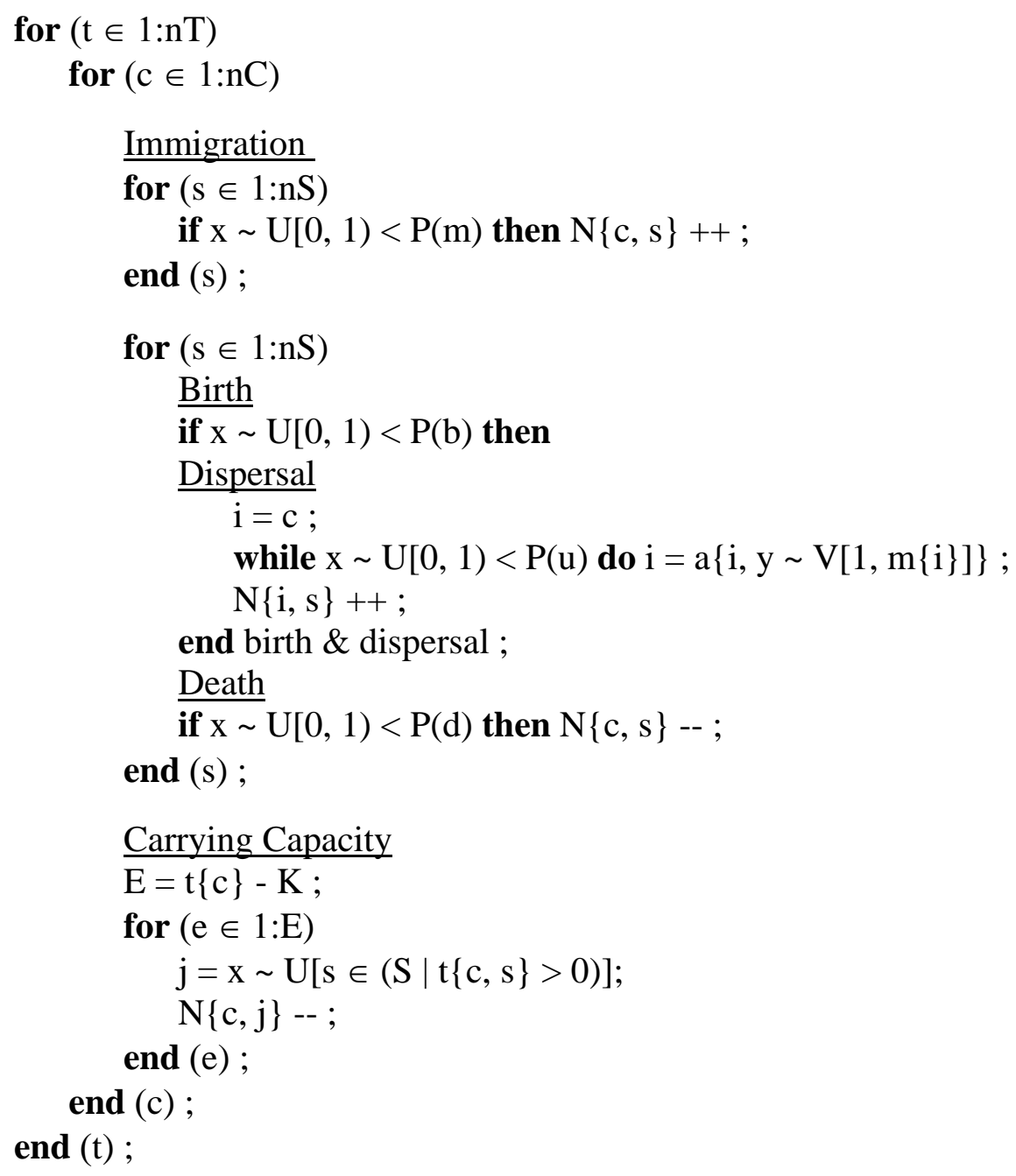

where $\mathrm{t}$ indexes time steps, $\mathrm{nT}$ is the total number of time steps, : is the range operator (a:b means integers between a and $b$ ), $c$ indexes communities, $\mathrm{nC}$ is the total number of communities, $\mathrm{s}$ indexes species, $\mathrm{nS}$ is the number of species, $\mathrm{x}$ is a random number, $\mathrm{U}[\mathrm{a}, \mathrm{b})$ is the uniform continuous distribution from the closed lower bound a to the open upper bound $\mathrm{b}, \mathrm{P}(\mathrm{m})$ is the probability of immigration, $\mathrm{N}\{\mathrm{c}, \mathrm{s}\}$ is the population of species $\mathrm{s}$ in community c, ++ is the increment operator (by one unit), $\mathrm{P}(\mathrm{b})$ is the probability of birth, i indexes community location, $\mathrm{P}(\mathrm{u})$ is the probability of dispersal, $\mathrm{a}\{\mathrm{i}$,$\} is the adjacency table of adjacent communities for$ community $\mathrm{i}, \mathrm{y}$ is a random variable, $\mathrm{V}[\mathrm{a}, \mathrm{b}]$ is the uniform discrete distribution for integers 
between a and $b, m\{i\}$ is the number of adjacent communities to community $i, P(d)$ is the probability of death, -- is the decrement operator (by one unit), $\mathrm{E}$ is the excess number of individuals in a community, $\mathrm{K}$ is the carrying capacity, e indexes the excess amount, $\mathrm{j}$ is a random species with population greater than zero.

The simulations used a set of 121 communities arranged either as an 11 by 11 tessellation of squares or as an 11 by 11 tessellation of hexagons. These tessellations were not connected across edges with toroidal topology but were considered as planar bounded spaces. Adjacency for square tessellations was defined by both the von Neumann neighborhood, meaning the side adjacent four neighbors, as well as the Moore neighborhood of those four plus the corner four neighbors.

The number of species was set to 15 , the number of individuals to 1000 , and the probability of immigration to 0.001 , for all simulation runs. The probability of birth and death was set to 0.3 ; and the probability of dispersal varied between 0.0 and 0.4 . There were 400 replicates, each having 2000 time steps, for each topology for each change in parameter settings.

Community outcomes were measured as the mean values of total community population at the end of each simulation, plus the community diversity measures of species richness, Shannon diversity, and Simpson diversity (the negative of the original measure, see Magurran 1988 for Shannon and Simpson measures and variants, and Lande 1996 for analysis of Simpson's measure). The formulas of the diversity measures, for one community, are:

Species richness: $\quad S=\sum_{i=1}^{\text {\#species }} I_{i}$

Shannon diversity: $\quad H^{\prime}=-\sum_{i=1}^{\# \text { species }} \frac{n_{i}}{N} \ln \frac{n_{i}}{N}$

Simpson diversity: $\quad D=1-\frac{\sum_{i=1}^{\# \text { species }} n_{i}\left(n_{i}-1\right)}{N(N-1)}$

where $I_{i}$ is the indicator variable having the value of 1 if there is at least one individual of species $i$, otherwise zero; $n_{i}$ is the number of individuals for species $i, N$ is the total number of individuals, and ln is the natural logarithm.

\section{Results}

For the three topologies in the configurations studied here, the mean number of adjacent communities was 5.29 for the hexagonal tessellation ("hexagon"), 3.64 for the square tessellation with the von Neumann neighborhood ("square-von Neumann"), and 6.942 for the square tessellation with the Moore neighborhood ("square-Moore"). 
Total populations at the end of the simulations had several characteristics (Figure 2). From relatively low ending populations at no dispersal all topologies reached a peak by a dispersal probability of 0.10 and then declined. Before 0.10 the ranking of values was square-von Neumann, hexagon, square-Moore. After 0.10 , the ranking was square-Moore, hexagon, squarevon Neumann. At 0.40 ending populations declined to between $40 \%$ and $60 \%$ of the highest values.

Median values of Shannon diversity had a less consistent pattern (Figure 3). Between minimum values for no dispersal and a probability of dispersal of about 0.20 , square-von Neumann had the highest diversity and hexagon was higher than square-Moore. Between dispersal probabilities of about 0.20 to 0.40 , hexagon had the highest values, although square-Moore was essentially the same from 0.36 to 0.40 . Median values of species richness and Simpson diversity had the same patterns.

Medians of Shannon diversity revealed one story but the distributions of these values revealed another aspect (Figures 4). Distributions of Shannon values for hexagon and square-Moore were generally similar whereas square-von Neumann was either skewed in the opposite direction (dispersals of 0.10 and 0.20 ) or broader in shape (dispersal of 0.30 ). All distributions had a strong negative skew at a dispersal probability of 0.40 , contrasting with more symmetric or positive skews at lower values of dispersal.

\section{Discussion}

Topological differences clearly show in the results in Figures 2, 3, and 4, but the patterns are complex. Ending populations have the simplest pattern, however even there the ranks of the topologies flipped at a low value of dispersal. As dispersal increased, the topologies with higher degrees of adjacency were less impacted by decline in population, possibly because of more even spread of populations.

It might be expected that topologies with more neighbors would lead to more evenly spread populations for each species also. The results for Shannon diversity only support this hypothesis for species diversity within communities at higher values of dispersal. At lower values, below the range 0.22 to 0.28 , the reverse was the case.

Behavior reversals were also evident in the distribution of Shannon diversity values. The hexagon topology had positively skewed histograms at dispersals of 0.10 and 0.30 but a nearly symmetric histogram at 0.20 . The square-von Neumann topology changed from strong negative skew at 0.10 , to a slight negative skew at 0.20 , to a broad distribution with slight positive skew at 0.30 , and then back to a strong negative skew at 0.40 . The square-Moore topology had positive skews between 0.10 and 0.30 , and then negative skew at 0.40 .

Although the results beg for further exploration and explanation, the dynamics of topological effects on properties of networks of ecological communities are complex and suggest potentially important issues. These impacts of topology on models of ecological processes have been little 
studied, and there is little awareness of the possibility of important effects. Because the large majority of modeling applications that use regular tessellations do use square tessellations, and because the two main topologies for implementing models using squares have drawbacks--von Neumann neighborhoods ignoring the diagonal neighbors, Moore neighborhoods implicitly assuming a non-tiling cell shape--greater attention to topological structures for process modeling is certainly called for.

Computer code in the $\mathrm{R}$ or $\mathrm{C}$ languages implementing the neutral model simulations are available from the first author.

\section{Acknowledgements}

This work benefited from discussions with the late Raymond O'Connor, as well as with Joshua Lawler, Scott Leibowitz, Brenda McComb, and Joan Baker. Helpful reviews by Ric Colasanti, Volker Bahn, and anonymous journal reviewers are appreciated also. The information in this document has been funded wholly (or in part) by the U.S. Environmental Protection Agency. It has been subjected to review by the National Health and Environmental Effects Research Laboratory's Western Ecology Division and approved for publication. Approval does not signify that the contents reflect the views of the Agency, nor does mention of trade names or commercial products constitute endorsement or recommendation for use.

\section{References}

Adler PB. 2004. Neutral models fail to reproduce observed species-area and species-time relationships in Kansas grasslands. Ecology 85(5):1265-1272.

Bell G. 2000. The distribution and abundance in neutral communities. The American Naturalist 155(5):606-617.

Bell G. 2001. Neutral macroecology. Science 293(28 September 2001):2413-2418.

Bell G. 2003. The interpretation of biological surveys. Proceedings of the Royal Society of London, B 270:2531-2542.

Bell G. 2005. The co-distribution of species in relation to the neutral theory of community ecology. Ecology 86(7):1757-1770.

Birch CPD. 2006. Diagonal and orthogonal neighbours in grid-based simulations: Buffon's stick after 200 years. Ecological Modelling 192:637-644.

Bithell M, Macmillan WD. 2007. Escape from the cell: spatially explicit modelling with and without grids. Ecological Modelling 200:59-78.

Brown JH. 2001. Toward a general theory of biodiversity. Evolution 55(10):2137-2138. 
Chase JM. 2005. Towards a really unified theory for metacommunities. Functional Ecology 19:182-186.

Chave J. 2004. Neutral theory and community ecology. Ecology Letters 7:241-253.

Chave J, Muller-Landau HC, Levin SA. 2002. Comparing classical community models: theoretical consequences for patterns of diversity. The American Naturalist 159(1):1-23.

Cioffi-Revilla C. 2002. Invariance and universality in social agent-based simulations. Proceedings of the National Academy of Sciences 99(Supplement 3):7314-7316.

Condit R, Pitman N, Leigh Jr EG, Chave J, Terborgh J, Foster RB, Núñez P, Aguilar S, Valencia R, Villa G, Muller-Landau HC, Losos E, Hubbell SP. 2002. Beta-diversity in tropical forest trees. Science 295(25 January 2002):666-669.

Conway JH, Sloane NJA. 1999. Sphere packings, lattices and groups, 3rd edition. SpringerVerlag, New York. 777 pp.

Enquist BJ, Sanderson J, Weiser MD. 2002. Modeling macroscopic patterns in ecology. Science 295(8 March 2002):1835-1836.

Etienne RS, Olff H. 2004. A novel genealogical approach to neutral biodiversity theory. Ecology Letters 7:170-175.

Flache A, Hegselmann R. 2001. Do irregular grids make a difference? Relaxing the spatial regularity assumption in cellular models of social dynamics. Journal of Artificial Societies and Social Simulation 4(4). URL: http://www.soc.surrey.ac.uk/JASSS/4/4/6.html.

Frisch U, Hasslacher B, Pomeau Y. 1986. Lattice-gas automata for the Navier-Stokes equations. Physical Review Letters 56(14):1505-1508.

Gilbert B, Lechowicz MJ. 2004. Neutrality, niches, and dispersal in a temperate forest understory. Proceedings of the National Academy of Sciences 101(20):7651-7656.

He F. 2005. Deriving a neutral model of species abundance from fundamental mechanisms of population dynamics. Functional Ecology 19:187-193.

Hubbell SP. 2001. The unified neutral theory of biodiversity and biogeography. Princeton University Press, Princeton, New Jersey. 375 pp.

Hubbell SP. 2005. Neutral theory in community ecology and the hypothesis of functional equivalence. Functional Ecology 19:166-172.

Keller JK, Heimbuch D, Richmond M. 1979. Optimization of grid cell shape for analysis of wildlife habitat. Proceedings of Proceedings of the Thirteenth International Symposium on 
Remote Sensing of Environment, Vol. III. Environmental Research Institute of Michigan, Ann Arbor. pp. 1419-1428.

Lande R. 1996. Statistics and partitioning of species diversity, and similarity among multiple communities. Oikos 76(1):5-13.

Magurran AE. 1988. Ecological diversity and its measurement. Princeton University Press, Princeton, New Jersey. 179 pp.

Magurran AE. 2005. Species abundance distributions: pattern or process? Functional Ecology 19:177-181.

McGill B, Collins C. 2003. A unified theory for macroecology based on spatial patterns of abundance. Evolutionary Ecology Research 5:469-492.

McGill BJ. 2003. A test of the unified neutral theory of biodiversity. Nature 422(24 April 2003):881-885.

McKane AJ, Alonso D, Solé RV. 2004. Analytic solution of Hubbell's model of local community dynamics. Theoretical Population Biology 65:67-73.

Murray Jr BG. 1967. Dispersal in vertebrates. Ecology 48(6):975-978.

Nee S. 2005. The neutral theory of biodiversity; do the numbers add up? Functional Ecology 19:173-176.

Nee S, Stone G. 2003. The end of the beginning for neutral theory. Trends in Ecology and Evolution 18(9):433-434.

Olea RA. 1984. Sampling design optimization for spatial functions. Mathematical Geology 16(4):369-392.

Stauffer D, Aharony A. 1992. Introduction to percolation theory, 2nd edition. Taylor \& Francis, London. 181 pp.

Toffoli T, Margolus N. 1987. Cellular automata machines. MIT Press, Cambridge, Massachusetts. 259 pp.

Vallade M, Houchmandzadeh B. 2003. Analytical solution of a neutral model of biodiversity. Physical Review E 68(061902):1-5.

Volkov I, Banavar JR, Hubbell SP, Maritan A. 2003. Neutral theory and relative species abundance in ecology. Nature 424(28 August 2003):1035-1037. 
Wolfram Mathworld. 2006. Percolation threshold. URL:

http://mathworld.wolfram.com/PercolationThreshold.html. Accessed 18 January 2006.

Yu DW, Terborgh JW, Potts MD. 1998. Can high tree species richness be explained by Hubbell's null model? Ecology Letters 1:193-199. 


\section{Captions}

Figure 1. Topologies analyzed in the paper are (a) hexagons, (b) squares with the von Neumann neighborhood, and (c) squares with the Moore neighborhood. Some cellular automata applications use (d) squares with the Margolus neighborhood in order to achieve hexagon neighborhood properties.

Figure 2. Mean for all simulations of total populations per community across all species at the end of the 2000 step replicates, for each dispersal probability, by topology. Dispersal probabilities were from 0.00 to 0.40 by increments of 0.05 .

Figure 3. Median values of Shannon diversity across all simulations and communities, for each dispersal probability, by topology. Dispersal probabilities were from 0.00 to 0.40 by increments of 0.02. The graphs are lowess smoothed versions of the point by point trajectories, where the proportion of points used for each smoothed value was 0.3 of the total.

Figure 4. Histograms of the distribution of Shannon diversity values for each combination of dispersal probability and topology. Each histogram includes 48,400 values. 
(a) Hexagon Tessellation, Hexagon Neighborhood

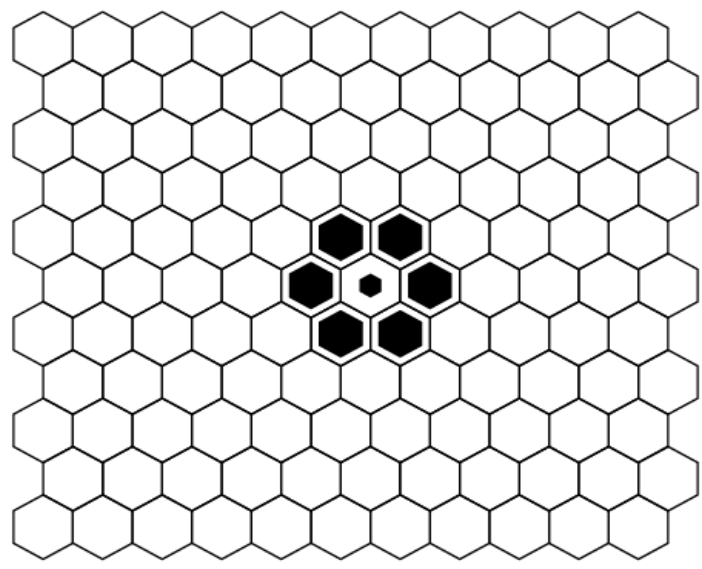

(c) Square Tessellation, Moore Neighborhood

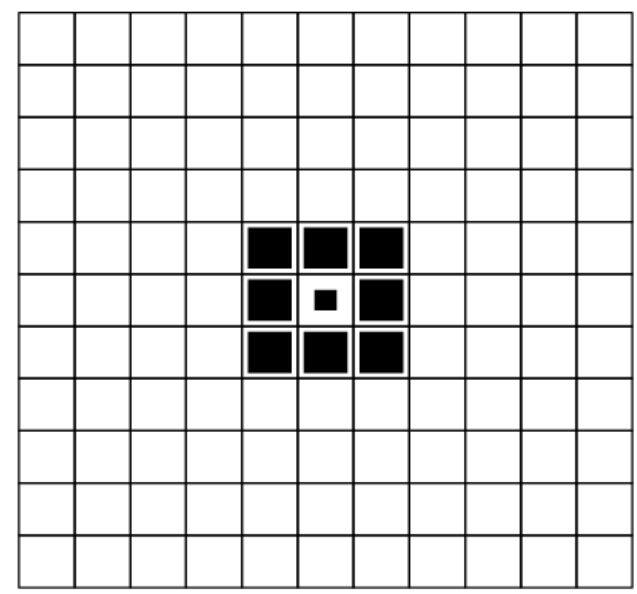

(b) Square Tessellation, von Neumann Neighborhood

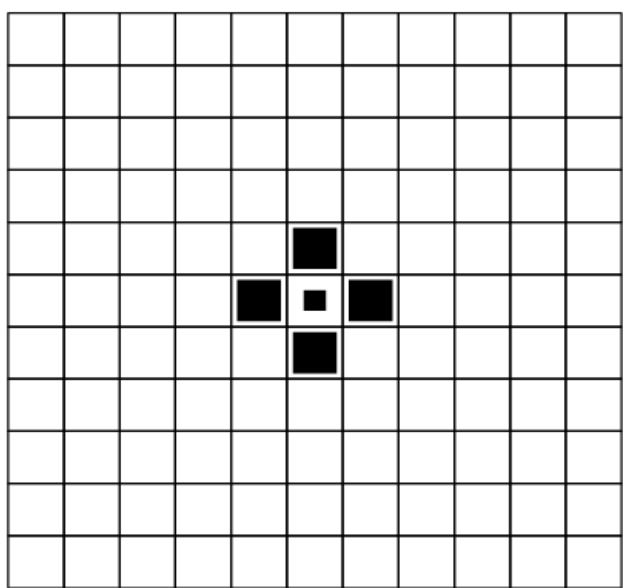

(d) Tilted Square Tessellation, Margolus Neighborhood

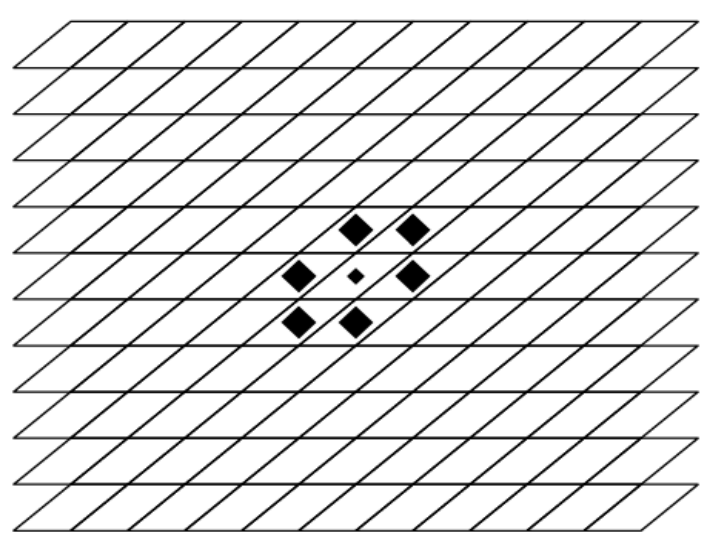

Figure 1 


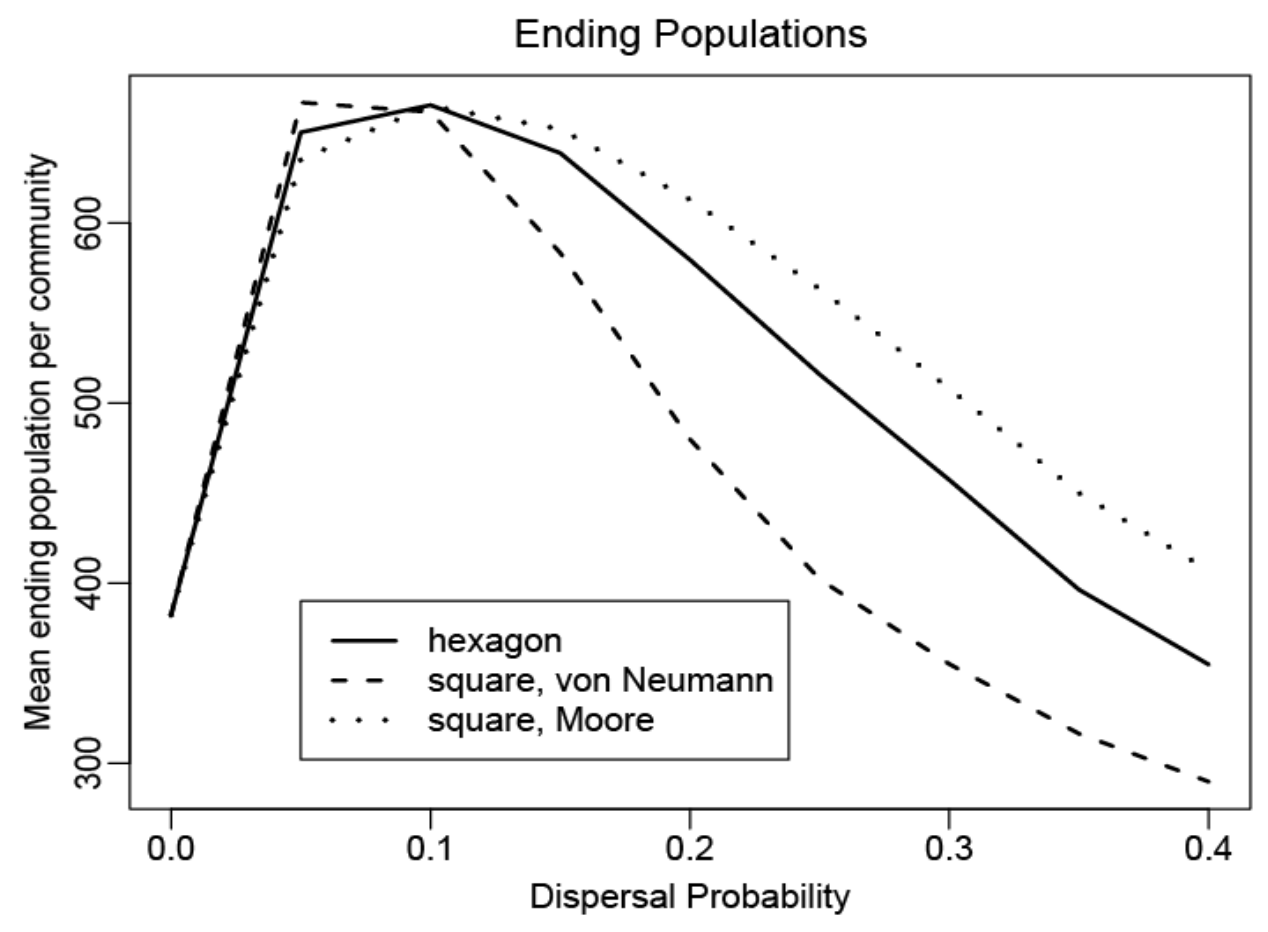

Figure 2 


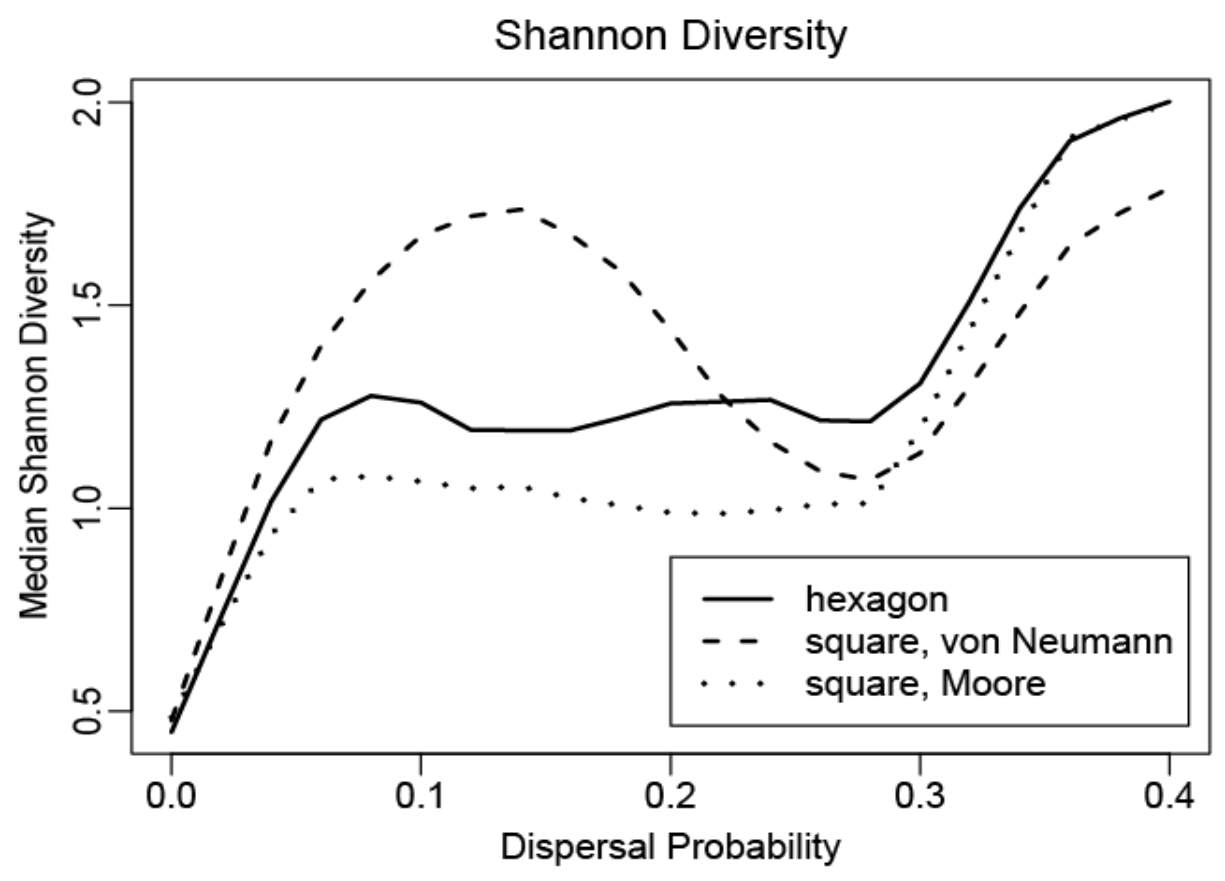

Figure 3 


\section{Shannon Diversity}
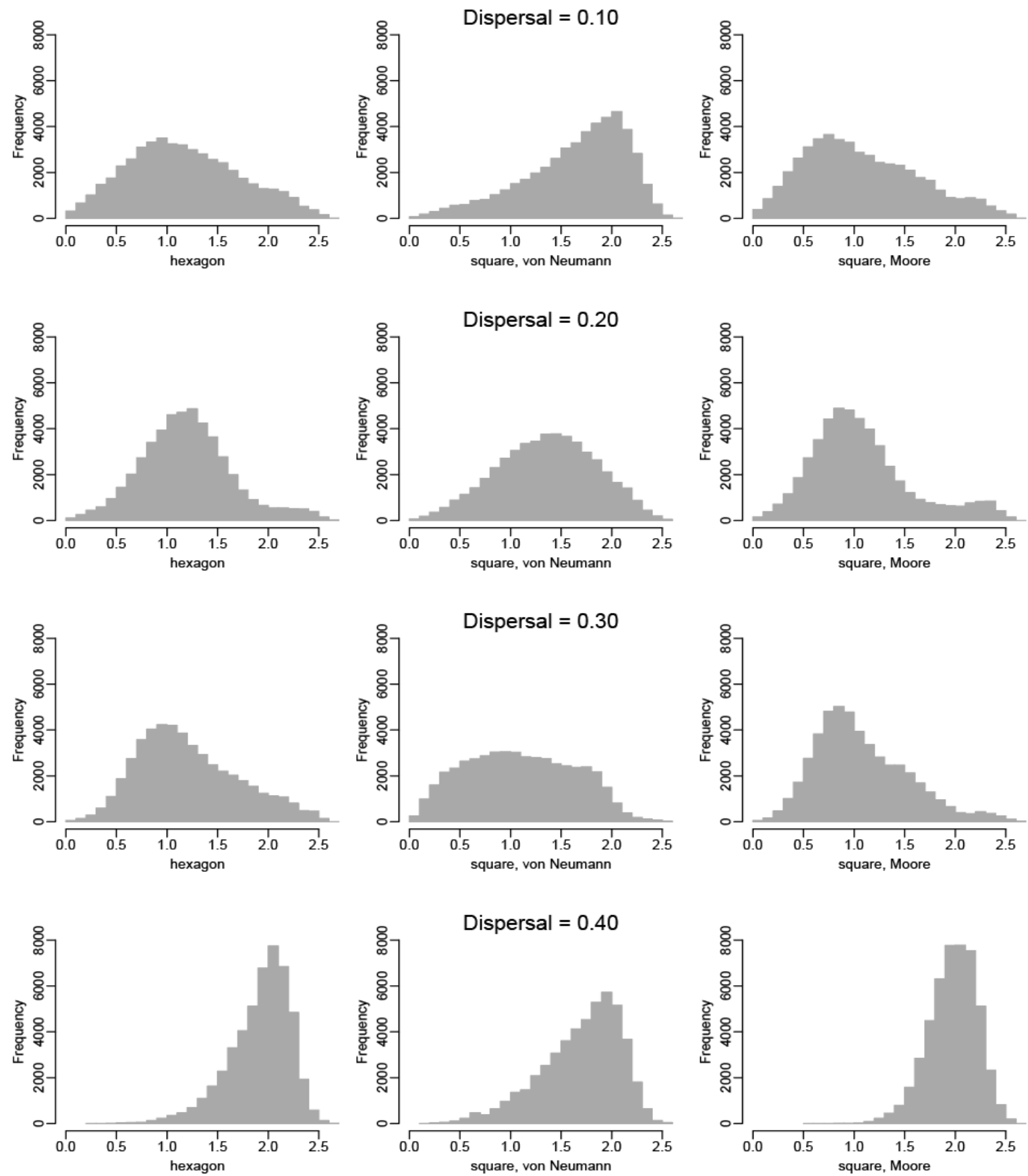

Figure 4 